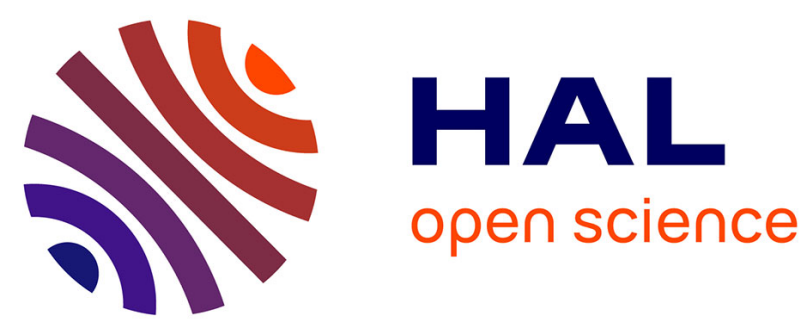

\title{
Du pluralisme dans la science
}

Nicolas Bouleau

\section{To cite this version:}

Nicolas Bouleau. Du pluralisme dans la science. 2008. halshs-00374576

\section{HAL Id: halshs-00374576 \\ https://shs.hal.science/halshs-00374576}

Preprint submitted on 9 Apr 2009

HAL is a multi-disciplinary open access archive for the deposit and dissemination of scientific research documents, whether they are published or not. The documents may come from teaching and research institutions in France or abroad, or from public or private research centers.
L'archive ouverte pluridisciplinaire HAL, est destinée au dépôt et à la diffusion de documents scientifiques de niveau recherche, publiés ou non, émanant des établissements d'enseignement et de recherche français ou étrangers, des laboratoires publics ou privés. 
Faites-le pour le bien de tous, pour redonner à tous la tranquillité perdue ! Ils veulent une vérité, peu importe laquelle, pourvu qu'elle soit précise et irrécusable ! Eh bien, donnez-leur ce qu'ils demandent !

Chacun sa vérité, Luigi Pirandello.

\section{La pluralité des mondes...}

Lorsque dans les années 1830, un mathématicien hongrois envoya à Carl Friedrich Gauss, le mémoire établi par son fils Janos Bolyai (1802-1860) sur la géométrie sans l'axiome des parallèles d'Euclide, Gauss lui répondit qu'il connaissait cela depuis plus d'une trentaine d'année déjà. Et en effet, on a trouvé dans ses manuscrits des travaux sur le sujet rédigés dès 1792. Il en était venu à la conviction qu'il était possible, en niant l'axiome des parallèles, de développer "une étrange géométrie tout à fait différente de la nôtre [...] entièrement cohérente en elle-même" où l'on pouvait "résoudre analytiquement tout problème, à la détermination près d'une constante" ${ }^{1}$. Pour quelle raison n'avait-il rien publié ?

Il avait peur, tout simplement. Dans une lettre à son collègue Bessel de 1829, il évoque cette géométrie non-euclidienne, comme il l'appelle, et écrit "j'appréhende les clameurs des Béotiens si je voulais exprimer complètement mes vues".

C'est un monde qui s'effondre. Pour le comprendre, il faut abandonner la vision actuelle de ces questions. Comme l'a souligné Hélène Metzger en de belles pages ${ }^{2}$, on ne peut saisir ce que l'histoire apporte à la philosophie des sciences si l'on décrit les énigmes du passé avec les solutions d'aujourd'hui.

La découverte faite par Gauss, Bolyai et Lobatchevski est, beaucoup d'auteurs l'ont remarqué, en contradiction flagrante avec la philosophie kantienne qui considère les mathématiques comme des jugements synthétiques a priori, et qui confère ainsi une place absolue à la géométrie. Mais bien plus profondément me semble-t-il, ce sont les bases des relations entre la science et la civilisation héritées des Grecs qui se fissurent. A bien des égards, les Grecs pensaient que la géométrie — qu'ils mettaient en œuvre dans leurs temples et dans les tracés de leurs villes nouvelles - était de leur côté, du côté de la civilisation contre les forces sauvages de la nature et des barbares. La vue des ruines labyrinthiques des palais crétois les effrayait, leur faisant imaginer les pires monstruosités. Et Vitruve, avant d'aborder les proportions et les mesures des édifices se réfère aux Grecs en racontant que "le philosophe Aristide, disciple de Socrate, s'étant sauvé d'un naufrage sur les côtes de l'île de Rhodes, et ayant aperçu des figures géométriques tracées sur le sable, dit en s'écriant à ceux qui étaient avec lui : ne craignons rien je vois des traces d'hommes !".

Qu'il puisse exister plusieurs rationalités géométriques différentes et non la géométrie, la vraie, celle d'Euclide, est un drame de la raison. Elle est d'une nature différente des ruptures dues à l'héliocentrisme (Copernic), à l'évolution (Darwin) et à l'inconscient que Freud appelle des blessures narcissiques qui restent compatibles avec une science univoque. Elle fut traitée d'abord par le secret ou la confidentialité, puis suscita un énorme travail, durant tout le 19 ème siècle, pour tenter de commercer avec la région découverte par des exemples, des traductions partielles (Cayley, Beltrami, Riemann, Felix Klein) et elle finit, par accoutumance et familiarité, à être considérée comme une des régions ordinaires du savoir mathématique.

\footnotetext{
${ }^{1}$ C. F. Gauss, Werke, 12 vol. Göttingen, 1870-1927, t8, p165 et seq.

${ }^{2}$ H. Metzger, La méthode philosophique en histoire des sciences (1914-1939), Fayard 1987.
} 
Les mathématiques japonaises de la période de l'Edo (1603-1867) nous montrent un autre cas d'émergence de la pluralité scientifique : il ne s'agit plus de bifurcation dans le développement internaliste des contenus mais de circonstances purement socio-politiques qui coupèrent complètement le Japon du monde occidental. Durant cette longue période d'isolement, des gens de toutes conditions sociales, du paysan au samouraï produisirent des théorèmes de géométrie remarquablement différents de ceux produits à l'Ouest. Ces théorèmes n'étaient pas publiés dans des livres, mais dessinés, avec souvent de belles couleurs, sur des tablettes de bois suspendues sous le toit à l'entrée des temples ou des autels. La méthode de découverte des théorèmes consistait en une concentration intense et prolongée — des mois, parfois des années — sur une figure géométrique soigneusement tracée ${ }^{3}$. Donc une pratique du raisonnement non discursive mais contemplative et un usage social de la science très éloigné de la fabrication de machines ou de dispositifs.
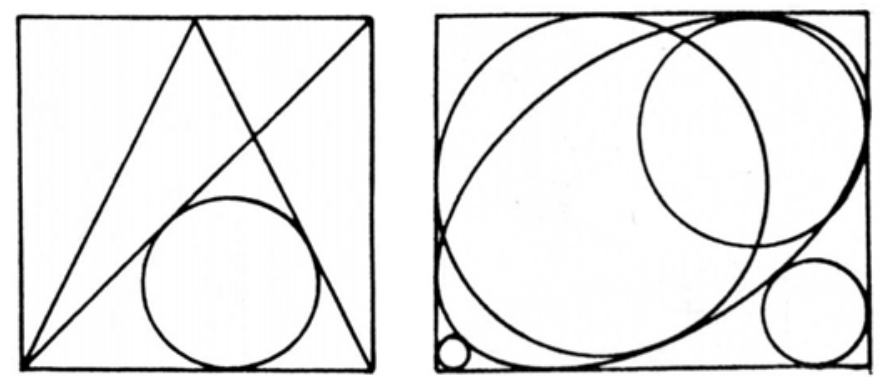

Il peut s'agir de problèmes simples comme ici à gauche d'exprimer le rayon du cercle en fonction du côté du carré (tablette de 1877 à Miyagi) ou difficiles comme à droite où il faut trouver la relation entre les quatre rayons des cercles tangents aux côtés du rectangle et à l'ellipse (tablette de 1830 à Yamagata), parfois très savants faisant intervenir des équations de degré élevé.
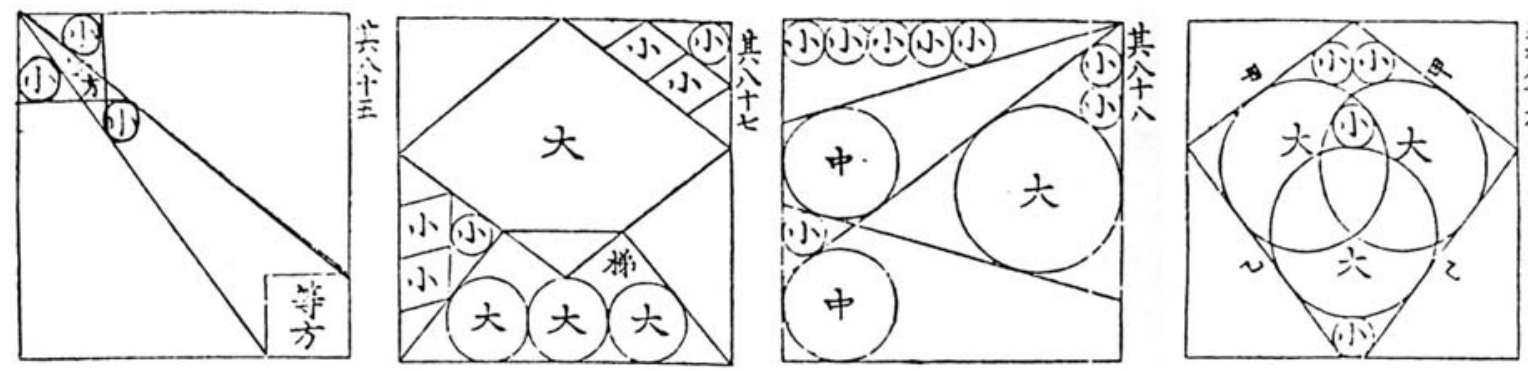

Difficile d'imaginer ce qu'aurait pu fournir cette branche de la connaissance si elle s'était davantage développée.

Avec l'approfondissement de la méthode axiomatique et l'essor de la logique au tournant des 19 et $20^{\text {ème }}$ siècles, on peut dire que le vertige créé par les géométries noneuclidiennes se renouvelle cette fois pour l'ensemble des mathématiques : il y a plusieurs interprétations, c'est-à-dire plusieurs façons de les comprendre. L'une d'elle a pris la force d'une véritable dissidence, c'est l'analyse non-standard, mais la rupture épistémologique remonte — avant Gödel — au théorème de Löwenheim et Skolem (1915-1920) qui établit que toute théorie du langage des prédicats du premier ordre (le langage usuel des mathématiques) qui a un modèle a un modèle dénombrable. Comme les nombres réels au sens ordinaire ne sont pas dénombrables, cela veut dire qu'il y a plusieurs Analyses, plusieurs Arithmétiques, etc. L'analyse non-standard a aujourd'hui un tableau de chasse tout à fait honorable, y compris des retombées sur des problèmes formulés en termes classiques concernant les équations différentielles dépendant d'un paramètre ou le calcul stochastique.

\footnotetext{
${ }^{3}$ Cf. San Gaku, Japanese Temple Geometry Problems, Winnipeg, Canada, 1989.
} 
En physique la longue histoire des conceptions ondulatoire et corpusculaire de la lumière débouche au $20^{\text {ème }}$ siècle sur la dualité ondes-corpuscules de la mécanique quantique $^{4}$. La contradiction a progressivement été promue, dans des cadres théoriques de plus en plus sophistiqués, au rang de "propriétés duales d'un même phénomène" où les mystères de la "réduction du paquet d'onde" prennent, comme plusieurs autres interrogations naturelles, le statut de questions mal posées en tant qu'exprimées selon une intuition macroscopique inadaptée. Il n'en reste pas moins que le formalisme quantique donne lieu à plusieurs interprétations utiles au sein même des calculs quantiques : l'espace de Fock possède deux interprétations probabilistes simples et tout à fait distinctes, celle du processus de Wiener et celle des systèmes poissoniens de particules.

Les dualités sont moins inquiétantes que les pluralités. Elles se répondent et l'on s'en accommode finalement, à la manière de Hegel, en admettant que leur rivalité engendre historiquement une réalité d'ordre supérieur. Penser deux interprétations à la fois est difficile, mais nous sommes bien obligés de nous résoudre finalement à l'idée qu'une vision duale de la nôtre a droit de cité car nous sommes en permanence confrontés à cette question philosophique sans solution de savoir si les hommes et les femmes, ces animaux qui n'ont pas les mêmes chromosomes, pensent de la même façon ${ }^{5}$. Une autre raison tient à ce que les dualités sont la première étape dans la plupart des tentatives de compréhension. Ceci fut noté par Saussure au niveau du langage, et plusieurs sociologues considèrent que l'individu se construit par la langue, par l'éducation et par l'insertion économique de sorte que sa personnalité la plus intime, ses valeurs les plus hautes sont en partage et/ou en opposition avec celle de sa communauté.

Au demeurant, le bilan de l'étude de la charpente logique des sciences est que les formalismes ont plusieurs interprétations, plusieurs sens. On ne peut pas séparer dans la science ce qui est rigoureux, absolu, universel et objectif, fondé sur des définitions précises, sur des protocoles exhaustifs et sur la déduction logique, de ce qui est relatif aux signifiés, aux résonances émotives nées socialement et historiquement, ni en physique par des règles de correspondance comme l'espéraient les néo-positivistes, ni en mathématiques en les voyant comme purement formelles car on ne sait les faire avancer qu'en les manipulant par le sens. Nous devons admettre que la théorie (l'étude logique des formalismes) nous dit qu'il y a pluralité d'interprétations possibles. Aussi la question philosophique se présente selon l'alternative suivante : ou bien ce pluralisme est susceptible d'imprégner toutes les relations de la science et de la technique avec la vie économique et sociale, et donc peut — ou risque de - se renforcer par la diversité des ancrages politiques et des intérêts divergents des acteurs ; ou bien un contenu positif peut être préservé qui ne dépend pas de ces diverses interprétations, véritable travail des scientifiques qui peut être mené indépendamment de la culture, des intérêts économiques et sociaux et de tout ce qui fait que certains mots plutôt que d'autres sont pour eux source de motivation.

\section{La modélisation et le pluralisme}

Cette alternative s'éclaire considérablement si l'on considère une pratique venant prendre place précisément dans l'entre deux science-société qui s'est développée de façon fulgurante durant le troisième tiers du $20^{\text {ème }}$ siècle, et donne quelques rides aux analyses classiques des relations à la technique, y compris au concept de techno-science, je veux parler de la modélisation et particulièrement de la modélisation du contexte, c'est-à-dire la

\footnotetext{
4 Cf. J.-M. Lévy-Leblond, Aux contraires, Gallimard 1996; M. Bitbol, Mécanique quantique, Flammarion 1999.

${ }^{5}$ Cf. Carol Gilligan, A different voice, Harvard Univ.Press 1982.
} 
modélisation de l'environnement, ou, pour un agent économique, la modélisation du cadre de son action.

Alors qu'avec Newton et la gravitation, avec Maxwell et l'électromagnétisme, avec Einstein et les relativités restreinte et générale, etc., la façon la plus efficace d'embrasser un très grand nombre de phénomènes était de disposer d'une théorie - ce qui fait que l'épistémologie s'est focalisée sur les théories - nous sommes maintenant capables, avec une précision et une puissance de stockage croissantes, d'archiver des données fortuites, prises en mesurant le monde qui nous entoure, qui viennent servir de point de départ à des projections, évaluations, projets divers, très utiles en pratiques. D'un côté — cela est noté par plusieurs auteurs - il semble que l'invention de théories s'essouffle quelque peu, d'un autre côté, les théories existantes se trouvent démultipliées dans leurs applications par la modélisation et celle-ci a, en plus, l'audace — ou l'avantage — d'employer les mêmes moyens empathiques que le langage ordinaire pour convaincre et défendre à l'occasion les vues de tel ou tel acteur économique ou tel groupe décisionnel. On arrive à une véritable langue, avec laquelle on peut dire le meilleur comme le pire, qui use de symbolisme comme les sciences et d'embrayages, de litotes et autres analogies comme Bossuet, Marx ou Mallarmé.

Corrélativement, la modélisation a permis de pointer les faiblesses de la philosophie de Karl Popper dans son ambition d'établir une césure nette entre ce qui est scientifique et ce qui ne l'est pas. Dans bien des situations courantes - et dans toutes les études prospectives - on est en face de plusieurs lectures des faits expérimentaux qui constituent autant de théories compatibles avec les données disponibles mais porteuses de conséquences distinctes dans l'avenir proche ou lointain. Ces théories - l'exemple de la modélisation des hauteurs d'eau d'une rivière est ici générique ${ }^{6}$ — sont chacune un cadre de pensée plutôt qu'un système réfutable par une expérience décisive. Elles sont une manière de prendre les choses et restent indéfiniment perfectibles si de nouvelles données sont recueillies. L'attitude qui consisterait à les rejeter en bloc comme non-scientifiques au profit d'une théorie qui, elle, disposerait d'une expérience cruciale (mais dont la réponse est ajournée) est intenable parce que de telle théories réfutables sont elles-mêmes nombreuses et peuvent orienter le décideur dans des directions imprudentes, dangereuses ou même démentes.

Si à l'exemple des hauteurs d'un cours d'eau nous substituons l'étude du marché potentiel d'un prototype innovant par une firme sur la base de données statistiques et de modélisation économique et financière, nous retrouvons l'évidence de bon sens que la représentation dépend de l'entité sociale qui l'élabore, des informations dont elle dispose, des objectifs qu'elle poursuit et des moyens qu'elle peut mettre en œuvre, autant de connaissances subjectives qui expliquent que, sur les marchés financiers, les actifs incertains, au lieu de prendre une simple décote à cause de cette incertitude, voient leur cours s'agiter au gré d'acheteurs qui les pensent prometteurs et de vendeurs qui s'en méfient.

\section{Force et contradictions du monisme}

Fondé sur le trépied a) du progressisme conquérant dont l'histoire remonte à Bacon et qui, après les lumières, les saint-simoniens, etc., trouve une nouvelle dynamique en remède aux morosités dues à la dégradation de l'environnement, b) du libéralisme économique maintenant mondialisé qui, touchant les limites des ressources naturelles, se focalise de plus en plus sur l'engouement suscité par l'innovation technique, c) d'un néo-darwinisme généralisé qui contribue à justifier des faits accomplis des forts contre les faibles, s'installe un positivisme revigoré, qui prolonge celui de Comte et de Mill, en allant plus loin même que les

\footnotetext{
${ }^{6}$ Cf. par exemple, N. Bouleau, Philosophies des mathématiques et de la modélisation, p. 307 et seq. Le cœur de l'argument est qu'on ne dispose jamais que d'un nombre fini de points de mesure.
} 
vues les plus passionnées de Renan ou de Teilhard de Chardin par l'enjeu nouveau des anthropotechniques.

Parmi les racines historiques récentes de ce monisme contemporain, on doit citer certainement les liens dégagés par Max Weber entre la philosophie d'entreprendre, au cœur du capitalisme, et le concept de la grâce du protestantisme (beruf chez Luther et prédestination chez Calvin). Mais je pense que tout le courant de la philosophie analytique (donc aussi celui du pragmatisme qui lui est intimement lié) entretient une ambiguïté qui profite au monisme progressiste. C'est ce qu'on peut appeler le préjugé de supériorité analytique, qui est une forme de logicisme conduisant à l'intolérance. Il consiste en l'argument suivant : "si ce que je crois est vrai, alors les autres croyances sont n'importe quoi". Non seulement elles sont fausses mais elles sont arbitraires et je ne leur donne même pas le droit d'une charpente cohérente. Le ex falso sequitur quodlibet de la logique aristotélicienne imprègne notre vision de la connaissance. C'est cette règle qui fait que les théories contradictoires sont sans intérêt et qui donne de la valeur aux énoncés démontrés, à savoir les théorèmes. Mais en toute généralité ce principe s'appuie sur une idée de la vérité bien trop forte. Il étend abusivement (naïvement ?) à ce qui relève du sens une règle purement analytique. Cette extension est pleine de sous-entendus commodes pour disqualifier tout adversaire : il y a une pensée (la nôtre) qui possède à la fois assez de contenu pour asseoir la morale et suffisamment proche de la forme analytique pour en tirer sa force d'exclusion. Sa première conséquence est de confondre pluralisme et relativisme.

Il convient de rappeler que la logique ne confond en aucun cas ce qui relève des règles de démonstration et ce qui relève des interprétations, i.e. de la théorie des modèles. Les modèles utilisés pour établir les résultats d'indépendance de certains axiomes, le modèle des ensembles constructibles de Gödel, ou ceux de Cohen construits à l'aide d'éléments génériques, ne sont évidemment pas n'importe quoi. Dans les écritures formalisées les symboles d'implication et de satisfaction ne sont pas les mêmes, les relations des modèles entre eux ne relèvent pas de règles régissant les énoncés ${ }^{7}$.

Il y a une variété d'interprétations des mathématiques, mais, en pratique, on n'en utilise que quelques-unes, dont l'interprétation non-standard d'Abraham Robinson, qui, il faut insister, n'est pas du tout n'importe quoi. De même plus localement on peut donner trois ou quatre interprétations de la théorie du potentiel (gravitation, électrostatique, chaleur, probabiliste, etc.) mais guère plus.

Vouloir à tout prix confondre pluralisme et relativisme est la principale stratégie offensive du positivisme.

Auguste Comte ne prend pas la peine de réfuter les systèmes de pensée différents du sien, il y a l'homme à l'état positif et l'homme à l'état primitif, c'est-à-dire sous le statut de "contradiction, cercle vicieux, spontanéité, résolution de l'insoluble" (sic). Pourquoi aujourd'hui encore dès qu'on parle de pluralisme en matière de connaissance est-ce une levée de boucliers ? On ressort la vieille machine de guerre qui consiste à confondre pluralisme et relativisme.

Prenons un exemple simple et actuel. Chaque crise financière conduisant à la variation rapide d'une devise par rapport aux autres peut faire l'objet de deux principales interprétations

\footnotetext{
7 Cf. J.-L. Krivine Théorie axiomatique des ensembles, PUF 1969; S. C. Kleene Logique mathématique, A. Colin 1971; P. J. Cohen Set theory and the continuum hypothesis, Benjamin 1966; et, pour une introduction P. Wagner La logique PUF 2007.

Le préjugé de supériorité analytique est implicite dans les nombreux travaux (D. Davidson "Paradoxes of irrationality"in R. Wollheim \& J. Hopkinds eds Philosophical Essays on Freud, Cambridge univ. Press 1982 p.289 et seq.; "Deception and Division" in E. Le Pore \& B. McLaughlin eds, Actions and Events, Perspectives on the Philosophy of Donald Davidson, Blackwell 1985 p. 138 et seq.) qui posent à l'infini la question de mieux cerner les contours de l'irrationalité des comportements et en arrivent toujours, comme les économistes, à la conclusion que si la frontière entre les comportements rationnels et irrationnels n'est pas évidente, en revanche il est clair que la discussion argumentée de la psychologie que "nous" menons fait, elle, partie de la rationalité.
} 
économiques. La première est celle des entreprises qui font leur bilan dans la dite devise. Considérant que la qualité de leur gestion n'est pas en cause puisqu'elle est la même qu'avant, elles concluront à une attaque spéculative. L'autre est celle des observateurs financiers extérieurs qui jugeront qu'il s'agit là d'un réajustement salutaire, la situation antérieure étant peu transparente ou mal informée. Chacune de ces deux interprétations peu de décliner dans tous les rouages de la description économique. Si l'on considère qu'il $\mathrm{y}$ a, en effet, des changements brusques qui sont interprétés par les uns comme des spéculations et par d'autres comme des réajustements, est-on relativiste pour autant ? Cela signifierait qu'on admet autant d'interprétations possibles cohérentes qu'il y a d'agents économiques, ce n'est pas la thèse avancée.

Considérer que la connaissance peut être plurielle, c'est accepter que les interprétations se regroupent en familles de Weltanschauungen, comme les opinions politiques se structurent en partis dans les systèmes parlementaires pluralistes. Faire entrer les sciences en démocratie ne peut se faire sans aller dans la direction de tels programmes ni sans la liberté des chercheurs et des laboratoires d'y adhérer explicitement et ouvertement.

Le monisme dont nous parlons est porteur d'une tromperie qu'il faut dénoncer dès aujourd'hui et que tentent de dissimuler les transhumanistes dans leur discours triomphaliste. Lorsque ceux-ci écrivent: "The ethical debates are like stones in a stream. The water runs around them. You haven't seen any biological technology held up for one week by any of this debates" $^{8}$, la morgue de ces universitaires convaincus de savoir qui va gagner se fonde uniquement sur la certitude qu'en système libéral, toute nouveauté, perçue par certains comme un avantage pour eux ou leur progéniture, trouvera un marché nonobstant toute considération morale et sera donc finançable. Mais cet automatisme, très fort en effet, n'est jamais qu'une propriété d'un système économique. Et dès lors qu'il est compris en tant que tel, il ne s'applique qu'à une science qui serait faite sans en tenir compte, le chercheur restant dans l'arrière-boutique.

Les enjeux de cette réflexion sont de penser les anthropotechniques avant qu'elles n'avancent par faits accomplis. Tout porte à croire qu'elles imposeront le pluralisme. La question est de savoir si ce pluralisme sera celui d'une scission de l'espèce humaine avec des individus "génétiquement nettoyés" qui ne se croiseront pas avec les autres, porteurs sains de gènes gênants, ou bien un pluralisme fondé sur la gestion politique étendu à la sphère technoscientifique permettant la coexistence démocratique d'une seule espèce représentée par des partis englobant valeurs, connaissances et techniques. Je renvoie ici à l'excellent livre de Francis Fukuyama Our posthuman future, Consequences of the biotechnology revolution ${ }^{9}$.

\section{Economisation et pluralisme.}

Pour me rapprocher de ce je présume être le cœur de ce colloque, je voudrais proposer maintenant quelques remarques sur le processus d'économisation, tentative d'élargir l'application de l'économie à de nouveaux domaines, en partant du cas historique des probabilités subjectives.

La théorie subjectiviste des probabilités considère que le calcul des probabilités s'applique aux croyances de chaque individu en présence d'aléa. Pour certains auteurs (Ramsey, de Finetti, Savage) elle est même la bonne façon de lui donner des fondements clairs et sans contradiction. Il s'agit alors de construire une "logique des croyances partielles"10 ${ }^{10}$ Les paradoxes tels que celui de St Petersbourg sont évités, grâce à l'idée que les joueurs cherchent à optimiser non l'espérance de leur gain mais l'espérance de l'utilité de leur

\footnotetext{
${ }^{8}$ J. Brockman, The New Humanists, Barnes \& Noble 2003, p. 228.

${ }^{9}$ Profile books, 2002.

10 "The subject of our inquiry is the logic of partial belief " F. P. Ramsey, "Truth and probability" in The foundations of Mathematics R. B. Braithwaite ed. Routledge \& Kegan Paul 1931.
} 
gain $^{11}$. Les fonctions d'utilité doivent satisfaire certaines conditions que l'on peut expliciter pour que le comportement du joueur soit cohérent, rationnel au sens précis d'être à l'abri de pertes évidemment exploitables par un autre joueur. Avec le fameux traité de J. Von Neumann et O. Morgenstern, la théorie des jeux et les fonctions d'utilité deviennent un moyen de justifier les liens entre les équilibres économiques et le comportement des agents, avancée considérable qui engendra de vastes prolongements. De Finetti et Savage ayant étudié comment l'agent reconsidère ses croyances en fonction des expériences qu'il rencontre, la théorie subjective donne naissance à ce qu'on appelle la théorie de la décision bayésienne, qui considère l'application des statistiques en partant au départ d'une probabilité a priori. En prenant ainsi le contre-pied de la doctrine objectiviste, on arrive souvent néanmoins à des résultats qui ne dépendent pas ou peu de la probabilité initiale qu'on avait choisie. D'où la fécondité de cette méthode qui permet des calculs plus aisés.

Je ne crois pas qu'il y ait véritablement querelle entre partisans de la théorie objective et ceux de la théorie subjective, si ce n'est dans les encyclopédies. Car en effet, quel est le propos des subjectivistes, quel est leur apport épistémologique ? Ils montrent que le calcul des probabilités "marche aussi" pour gérer les affaires d'un individu, avec les informations partielles dont il dispose sur le monde. Le champ d'application du calcul des probabilités est étendu. On voit tout de suite l'immense parti que peut en tirer la science économique : elle est mise au défi de dégager les lois économiques, les équilibres, déséquilibres, tendances, anticipations, à partir de comportements particuliers des agents, ceux-ci étant seulement supposés gérer de façon cohérentes leurs probabilités subjectives, de façon similaire à ce qu'avait fait la physique statistique à partir de l'hypothèse du mouvement désordonné des atomes. Le problème est ici plus complexe, mais le principe même de l'individualisme méthodologique, est fondé sur l'idée que chaque agent raisonne suffisamment pour qu'à son niveau la théorie subjective s'applique ${ }^{12}$.

Les contours de la théorie subjectiviste ne sont pas nets et bien des questions y restent en débat : certains choix ne semblent pas se faire conformément à une fonction d'utilité (paradoxe de Maurice Allais ${ }^{13}$ ) et nécessitent de conceptualiser une utilité plus abstraite dépendant éventuellement des propensions ou aversions au risque des agents. Ces controverses sont plutôt le signe d'une activité de recherche dynamique que de difficultés épistémologiques fondamentales.

La théorie subjectiviste pose une question philosophique d'une plus grande ampleur si on la considère en revanche sous un angle sociologique et politique. Les agents économiques sont rarement des individus mais des groupes de diverses puissances plus ou moins capables de recueillir et de traiter l'information. Elle laisse entendre que des connaissances strictement privées peuvent être pertinentes d'un point de vue logique et en cohérence avec les observations expérimentales effectuées. Elle vient alors confirmer et compléter ce que nous disions à propos de la modélisation. Une sorte d'équilibre de marché des agissements s'établit quoique les uns et le autres soient fondés sur des représentations différentes. Cela voudrait-il dire qu'une entité dominante pourrait emmener toute la science dans la direction subjective qui est la sienne?

Pour certains toutes les croyances, donc y compris les interprétations de la science, peuvent être traitées dans le cadre des probabilités subjectives. De quoi s'agit-il exactement ? C'est adopter le point de vue selon lequel "les individus peuvent quantifier leurs croyances, les ramener à une note selon un classement linéaire, même si telle croyance semble relever d'une pluralité de dimensions, car ils sont capables de l'évaluer par un pari monétaire dans la

\footnotetext{
${ }^{11}$ idée déjà suggérée par Daniel Bernoulli et Buffon qui écrit "Un mathématicien, dans ses calculs, n'estime l'argent que par sa quantité, c'est-à-dire sa valeur numérique, mais l'homme moral doit l'estimer par les avantages et les plaisirs qu'il peut lui procurer" cité par J. Bertrand Calcul des probabilités, 1888.

${ }^{12}$ Cf. K. Arrow, "Methodological individualism and social knowledge" The American Economic Review, 1994.

${ }^{13}$ Cf. L. J. Savage "Allais' paradox" in The foundations of Statistics, Dover 1972.
} 
loterie des actions concrètes qu'ils engagent". Une telle assertion — résumons-là en "tout est subjectivement probabilisable" — est impossible à démontrer. Certains phénomènes n'adviennent qu'une seule fois, qu'ils soient relatifs à un sujet particulier (le premier amour, la mort, etc.) ou universels (l'apparition des mammifères, la preuve de la conjecture de Goldbach, etc.), on ne peut donc établir cette assertion par des moyens statistiques, quant à l'obtenir par l'aveu des intéressés, les avis ne concordent pas. Il s'agit donc d'un principe, et, il peut y avoir des avantages et des inconvénients de l'adopter. Les avantages sont surtout d'ordre méthodologique pour les économistes, non seulement cela les amènent à un programme de recherche motivant qui consiste à retrouver à partir des seules hypothèses de rationalité des agents les lois des équilibres, ou des instabilités économiques, mais, avantage bien plus grand encore, cela repousse au delà de toute limite les frontières de leur discipline. Les inconvénients de ce principe sont ressentis par tous ceux qui pensent que certains choix, certains risques ne relèvent pas d'une négociation financière, et ne peuvent être pensés en faisant comme si c'était le cas. En fait, excepté pour les chercheurs en économie, et encore, ce principe apporte surtout des hésitations et perplexités supplémentaires dues - on ressent une certaine pression idéologique - à l'effort de penser comme si le principe était obligatoire.

Si je parcours rapidement par le souvenir les choix que la vie m'a amené à prendre, ils sont tous fondés sur le sens, la signification de ce qui se passe, je n'en vois aucun qui soit probabilisable, si ce n'est précisément des choix économiques dont la forme financière m'était imposée. Je parle des grandes décisions qui orientent le cours de l'existence, mais aussi des toutes petites choses : par exemple de tous les livres de ma bibliothèque, j'en ai parcouru beaucoup et je n'en ai lu complètement que quelques-uns, un choix à chaque fois absolument non probabilisable qui eut cependant une certaine importance pour la suite des événements me concernant...

Un exemple typique d'une situation non marchande où l'on est parvenu à installer des classements en copiant des raisonnements économiques de marché, est celui de la notation des chercheurs par le système des publications dans les revues plus ou moins réputées en fonction des citations des auteurs qui y publient. (cf. la méthodologie exposée par le web of science). Et ceci nous amène à relever un trait important de l'économisation : le rapprochement des extrêmes par repliement de l'échelle des valeurs.

Dans son article "De l'influence des péages sur l'utilité des voies de communication"14, considéré comme parmi les textes fondateurs du raisonnement économique, Jules Dupuit montre l'existence d'un niveau optimal de péage pour les ponts ou les barrières d'octroi en s'appuyant sur le fait que la courbe des recettes (trafic $x$ péage) en fonction du péage appliqué présente un maximum : elle part de zéro pour un péage nul, fait une bosse, et retourne en zéro pour des péages très élevés qui découragent tout trafic.

Ce phénomène de bosse est général. Dans le cas du web of science, la référence aux citations fait qu'on trouve dans la même catégorie les articles médiocres et les articles très au dessus du niveau courant. C'est tout à fait net en mathématiques, le "marché d'intérêt immédiat" des textes très difficiles est très réduit ${ }^{15}$. Dans les disciplines moins ésotériques cela se traduit par une pénalisation des travaux vraiment originaux, et de ceux qui sont

\footnotetext{
${ }^{14}$ Ann. des Ponts 207 (1849) 170-248.

15 David Ruelle raconte dans Hasard et chaos, O. Jacob 1991, à propos de son article "On the nature of turbulence" écrit avec Floris Takens, qui fut — c'est incontestable aujourd'hui — une rupture épistémologique au sens de Kuhn : "L'article expliquait pourquoi nous pensions que les vues de Landau sur la turbulence étaient fausses, et nous proposions quelque chose d'autre, faisant intervenir les attracteurs étranges [...] Nous avons soumis notre manuscrit à une revue scientifique appropriée, et il nous est rapidement revenu : refusé. L'éditeur n'aimait pas nos idées et nous renvoyait à ses propres articles pour que nous puissions apprendre réellement ce qu'était la turbulence [...] Finalement cet article a été publié dans une revue scientifique. (En fait, j'étais un des éditeurs de cette revue, et j'ai accepté moi-même l'article pour publication [...])".
} 
disciplinairement exportateurs, c'est-à-dire qui se fondent sur des savoirs extérieurs à la problématique telle qu'elle est majoritairement ressentie.

La formation d'une bosse est incontournable dans toute approche qui considérerait l'économie de la connaissance comme réductible à l'économie des biens ordinaires avec un seul paramètre scalaire qui serait la monnaie ou un substitut de la monnaie. Donc, prenant les territoires du titre de ce colloque au sens des contenus, nous sommes amenés à conclure qu'il faut absolument préserver, des territoires mal cartographiés, des jardins, des arrière-cours de l'esprit où sont concoctées des représentations jugées économiquement sans valeur. 